\title{
ITERATED SUFFICIENT M-OUT-OF-N (M/N ) BOOTSTRAP FOR NON-REGULAR SMOOTH FUNCTION MODELS
}

\author{
Beyaztas Ufuk ${ }^{1}$, Alin Aylin², Bandyopadhyay Soutir ${ }^{3}$ \\ ${ }^{I}$ Department of Statistics, Bartin University \\ ${ }^{2}$ Department of Statistics, Dokuz Eylul University \\ ${ }^{3}$ Department of Applied Mathematics and Statistics, Colorado School of Mines
}

\begin{abstract}
It is well known that under certain regularity conditions the boot- strap sampling distributions of common statistics are consistent with their true sampling distributions. However, the consistency results rely heavily on the underlying regularity conditions and in fact, a failure to satisfy some of these may lead us to a serious departure from consistency. Consequently, the 'sufficient bootstrap' method (which only uses distinct units in a bootstrap sample in order to reduce the computational burden for larger sample sizes) based sampling distributions will also be inconsistent. In this paper, we combine the ideas of sufficient and m-out-of-n $(\mathrm{m} / \mathrm{n})$ bootstrap methods to regain consistency. We further propose the iterated version of this bootstrap method in non-regular cases and our simulation study reveals that similar or even better coverage accuracies than percentile bootstrap confidence inter- vals can be obtained through the proposed iterated sufficient $\mathrm{m} / \mathrm{n}$ bootstrap with less computational time each case.
\end{abstract}

Key words: Asymptotic expansion, Bootstrap, Confidence interval.

\section{Introduction}

The nonparametric bootstrap method introduced by Efron (1979) has become a general framework to provide adequate solutions in a wide variety of statistical applications such as approximating the sampling distribution of a statistic, pre- diction, estimation, construction of confidence intervals, etc. The advantage of using the bootstrap method lies in the fact that under certain regularity condi- tions, the bootstrap sampling distribution of a statistic is consistent with the true sampling distribution, and it provides more accurate approximations compared to the first-order asymptotic theory. However, in some non-regular cases, it may become inconsistent due to the invalidity of such regularity conditions. The $\mathrm{m} / \mathrm{n}$ bootstrap which is based on replacing the bootstrap sample size $n$ with $m$ where $m=o(n)$ and $m \rightarrow \infty$ as $n \rightarrow \infty$, is a possible solution to regain the consistency of traditional bootstrap. See Atherya (1978), Bickel and Freedman (1981), Bre- tagnolle (1983), Swanepoel (1986), Beran (1997), and Wei et al. (2014) for more information. 
Recently, Singh and Sedory (2011) propose the sufficient bootstrap method that only uses the distinct units in a simple random sampling with replacement bootstrap sample of size $\mathrm{n}$ to reduce the computational burden and leads us to make better inferences in certain cases. Although it is consistent as long as the traditional bootstrap works, it becomes inconsistent in case of the inconsistency of the traditional bootstrap. As a solution, the idea of $\mathrm{m} / \mathrm{n}$ bootstrap may be combined with sufficient bootstrapping to regain the consistency, see for example Alin et al. (2017). In particular, let $\mathrm{X} 1, \mathrm{X} 2, \cdots$ is a sequence of independent and identically distributed (i.i.d.) random variables from an unknown distribution F.Also, let $\mathrm{x}_{\mathrm{n}}=\left(\mathrm{X}_{1}, \ldots, \mathrm{X}_{\mathrm{n}}\right)$ and $\mathrm{x}_{\mathrm{n}}{ }^{*}=$ $\left(\mathrm{X}_{1}{ }^{*}, \ldots, \mathrm{X}_{\mathrm{n}}{ }^{*}\right)$ be an i.i.d. random sample from $\mathrm{F}$ and the bootstrap resample, respectively. To perform sufficient $\mathrm{m} / \mathrm{n}$ bootstrap, we take a random sample of size $\mathrm{m}=\mathrm{o}(\mathrm{n}), \mathrm{x}_{\mathrm{m}}{ }^{*}=$ $\left(\mathrm{X}_{1}{ }^{*}, \ldots, \mathrm{X}_{\mathrm{m}}{ }^{*}\right)$, from $\mathrm{x}_{\mathrm{n}}$ but use only distinct observations. In this case, let $\mathrm{V}_{\mathrm{n}}{ }^{*}$ and $\mathrm{V}_{\mathrm{m}}{ }^{*}$ be the number of distinct observations in $\mathrm{x}_{\mathrm{n}}{ }^{*}$ and $\mathrm{x}_{\mathrm{m}}{ }^{*}$,respectively. Note that every unit in $\mathrm{x}_{\mathrm{n}}$ has probability $1-(1-1 / \mathrm{n})^{\mathrm{n}}$ to appear in a bootstrap sample. Consequently, it can be shown that the expected size of the usual and sufficient $\mathrm{m} / \mathrm{n}$ bootstrap resamples are approximately $\mathrm{E}\left(\mathrm{V}_{\mathrm{n}}{ }^{*}\right)$ $=1-\left(1-\mathrm{e}^{-1}\right)^{\mathrm{n}}$ and $\mathrm{E}\left(\mathrm{V}_{\mathrm{m}}{ }^{*}\right)=1-\left(1-\mathrm{e}^{-\mathrm{m} / \mathrm{n}}\right)^{\mathrm{n}}$, respectively.

Further, the iterated bootstrap method (see, Hall (1986)) can be useful in obtaining a higher degree of correction, for example to coverage accuracy, by using a second-level of bootstrap resamples to estimate and subsequently derive a correction for the coverage error in the original bootstrap procedure. Hall (1986), Beran (1987) DiCiccio and Romano (1988), and Hall and Martin (1988) provide theoretical properties of this method and prove that the iterating principle reduces the bootstrap errors in many statistical problems. In this study, we pro- pose a combination of the iterated bootstrap with sufficient and $\mathrm{m} / \mathrm{n}$ bootstrap methods (termed as iterated sufficient $\mathrm{m} / \mathrm{n}$ bootstrap) with an aim to reduce the coverage error of the percentile confidence interval in non-regular cases.

The rest of the paper is organized as follows. In Section 2, we provide a detailed information about the proposed method. In Section 3, the asymptotic expansions of the coverage probabilities for non-iterated and iterated sufficient $\mathrm{m} / \mathrm{n}$ bootstrap methods are given. To evaluate the finite-sample performance of the proposed method, we consider the non-regular case "function of means with null first-order differential" given by Shao (1994) and the results are also presented in Section 3. Finally, we conclude with some final remarks described in Section 4.

\section{Iterated sufficient $\mathrm{m} / \mathrm{n}$ bootstrap method}

Let $X_{1}, X_{2}, \ldots$ be a sequence of i.i.d. random variables from an unknown distribution $F \equiv F_{\theta}$, where the parameter $\theta$ is of our primary interest. Let $X_{1}, X_{2}, \ldots$ be a sequence of i.i.d. random variables from an unknown distribution $F \equiv F_{\theta}$, where the parameter $\theta$ is of our primary interest. Let $x_{n}=\left(X_{1}, \ldots, X_{n}\right)$ be an i.i.d. random sample from $F$, and let $R_{n}\left(x_{n}, \theta\right)$ be an approximately pivotal quantity whose distribution is given by $G_{n}=G_{n}(\cdot, F)$. In many cases of practical interest (e.g. a location-scale setting), the quantity $R_{n}\left(x_{n}, \theta\right)$ generally depends not 
only on the data and $\theta$, but also on nuisance parameter (such as a scale parameter $\sigma$ ). Suppose $\Theta$ is the set of all possible values of $\theta$. Then, a level $\alpha$ confidence set for the parameter $\theta$ can be obtained as

$$
\mathrm{S}_{n}=\left\{t \in \Theta: R_{n}\left(x_{n}, \theta\right) \leq G_{n}^{-1}(\alpha)\right\}
$$

for any given $\alpha \in(0,1)$, where $G_{n}{ }^{-1}(\alpha)$ describes the largest $\alpha$-th quantile of ttn. For any sequence $\{\mathrm{Fn}\}$ which converges to $F, G_{n}=G\left(\cdot, F_{n}\right)$ is supposed to converge weakly to a continuous distribution function $G=G(\cdot, F)$. Then, $G_{n}\left(R_{n}\left(x_{n}, \theta\right)\right)$ is distributed as uniform $U(0,1)$. In classical theory, $G_{n}$ is approximated by its limit. However, in most cases, it is not easy to obtain its limit when the estimate of the parameter is a complicated statistic. But bootstrap method makes it possible since it does not require the full knowledge of the underlying distribution. Let $\mathrm{x}_{\mathrm{n}}{ }^{*}=\left(\mathrm{X}_{1}{ }^{*}, \ldots, \mathrm{X}_{\mathrm{n}}{ }^{*}\right)$ be the bootstrap sample from $F_{n}$, where $F_{n}$ is the empirical distribution function which puts mass $1 / n$ to each data point. Let also $\hat{\theta}$ be the estimate of $\theta$ based on $x_{n}$. Then, the bootstrap analogue of $R_{n}\left(x_{n}, \theta\right)$ with the bootstrap distribution conditional on $x_{n}$ are given as $R_{n}{ }^{*}=R_{n}\left(x_{n}{ }^{*}, \hat{\theta}\right)$ and $G_{n}{ }^{*}=G_{n}{ }^{*}\left(\cdot, F_{n}\right)$, respectively. Similar to the Equation 1, the bootstrap estimate of $\mathrm{Sn}$ is obtained as

$$
\mathrm{S}_{n}{ }^{*}=\left\{t \in \Theta: R_{n}\left(x_{n}, \theta\right) \leq G_{n}{ }^{*-1}(\alpha)\right\}
$$

Since $F_{n}$ is a consistent estimate for $\mathrm{F}$, the bootstrap estimate $G_{n}{ }^{*}$ converges in probability to $\mathrm{G}$ as $\mathrm{n}$ increases. Moreover, $G_{n}{ }^{*}\left(R_{n}\left(x_{n}{ }^{*}, \hat{\theta}\right)\right)$ converges to a uniform $\mathrm{U}(0,1)$ distribution.

The iterating principle based on Beran (1987)'s prepivoting idea, transforms $R_{n}\left(x_{n}, \theta\right)$ into $R_{n, 1}\left(x_{n}, \theta\right)=G_{n}^{*}\left\{R_{n}\left(x_{n}, \theta\right)\right\}$ whose distribution is less dependent to $F$ compared to $G_{n}(x)$. Note that $R_{n, 1}\left(x_{n}, \theta\right)$ is exactly distributed $U(0,1)$ if $R_{n}\left(x_{n}, \theta\right)$ is the pivot. Let $G_{n, 1}(x)=$ $\mathrm{P}\left(\mathrm{R}_{\mathrm{n}, 1}\left(\mathrm{x}_{\mathrm{n}}, \theta\right) \leq \mathrm{x}\right)$ be the distribution of $\mathrm{Rn}, 1(\chi \mathrm{n}, \theta)$, and let $\mathrm{G}_{\mathrm{n}, 1}{ }^{*}(\mathrm{x})=\mathrm{P}\left(\mathrm{R}_{\mathrm{n}, 1}\left(\mathrm{x}_{\mathrm{n}}{ }^{*}, \widehat{\theta_{\mathrm{n}}}\right) \leq\right.$ $\left.\mathrm{x} \mid \mathrm{x}_{\mathrm{n}}\right)$ be its bootstrap estimate.

Then

$$
\mathrm{S}^{*}{ }_{n, 1}=\left\{t \in \Theta: R_{n}\left(x_{n}, \theta\right) \leq G_{n, 1}{ }^{*-1}(\alpha)\right\}=\left\{\theta: R_{n}\left(x_{n}, \theta\right) \leq G_{n}{ }^{*-1}\left[G_{n, 1}{ }^{*-1}(\alpha)\right]\right.
$$

defines $\alpha$ level iterated bootstrap confidence set for $\theta$. Generally, the error in $S_{n, 1}^{*}$ is smaller than the error in $\mathrm{S}_{n}^{*}$ and $S_{n}$. The iteration can be repeated continuously to reduce the coverage error of a confidence interval to a desired level. Martin (1990) shows that each iteration reduces the coverage error by an order of $n^{-1 / 2}$ and $n^{-1}$ for one-sided and two-sided intervals, respectively. On the other hand, each iteration increases the computation burden drastically. Also, at each nested resampling step, the support of the effective data set on which resampling is based drops - if there are $\mathrm{n}$ data points at level 1 , then there will be roughly $0.632 \mathrm{n}$ data points for level 2 resampling, $0.4 \mathrm{n}$ at $3 \mathrm{rd}$ level, $0.25 \mathrm{n}$ at 4 th level, and so on when the m-out-of-n bootstrap is used. Hence, by considering the shrinking support of the data and computational burden of this iterative procedure, to make our proposed method more practical and widely applicable we only recommend of doing the double bootstrap where iteration is only being done once. Let $x_{m, l}{ }^{* *}=$ $\left(X_{1}{ }^{* *}, \ldots, X_{l}{ }^{* *}\right)$ denotes a second level bootstrap sample with size 1 drawn randomly with replacement from $x_{m}{ }^{*}$. It also requires that $\mathrm{l}=\mathrm{o}(\mathrm{m})$ and $\mathrm{l} \rightarrow \infty$ as $\mathrm{m}, \mathrm{n} \rightarrow \infty$. Based on these, we perform our proposed method using the following steps. 
We start by drawing a simple random sample of size $\mathrm{m}, \mathrm{x}_{\mathrm{m}}^{*}$, with replacement from $\mathrm{x}_{\mathrm{n}}$. For the second level bootstrap, we draw another simple random sample of size 1 , denoted by $\mathrm{x}_{\mathrm{m}, \mathrm{I}}^{* *}$ with replacement from $\mathrm{x}_{\mathrm{m}}^{*}$, and use only distinct observations. Let $\mathrm{x}_{\mathrm{E}\left(\mathrm{V}_{\mathrm{m}}^{*}\right)}^{*}$ and $\mathrm{x}_{\mathrm{E}\left(\mathrm{V}_{\mathrm{m}, \mathrm{l}}^{* *}\right)}$ be the generic bootstrap resamples of size $\mathrm{E}\left(\mathrm{V}_{\mathrm{m}}^{*}\right)=\mathrm{n}\left(1-\mathrm{e}^{-\mathrm{m} / \mathrm{n}}\right)$ and $\mathrm{E}\left(\mathrm{V}_{\mathrm{m}, \mathrm{l}}^{* *}\right)=\mathrm{m}\left(1-\mathrm{e}^{-\mathrm{l} / \mathrm{m}}\right)$ from $\mathrm{x}_{\mathrm{n}}$ and $\mathrm{x}_{\mathrm{m}}^{*}$, respectively, both containing distinct elements only. Also let $\widehat{\theta}_{\mathrm{E}}^{*}\left(\mathrm{~V}_{\mathrm{m}}^{*}\right)$ and $\widehat{\theta^{* *}}{ }_{\mathrm{E}\left(\mathrm{V}_{\mathrm{m}, \mathrm{l}}^{* *}\right)}$ be the bootstrap estimators of $\theta \mathrm{n}$ and $\mathrm{R}_{\mathrm{E}\left(\mathrm{V}_{\mathrm{m}}^{*}\right)}^{*} \mathrm{R}\left(\mathrm{x}^{*} \mathrm{E}\left(\mathrm{V}_{\mathrm{m}}^{*}\right), \widehat{\theta_{\mathrm{n}}}\right)$ and $\mathrm{R}^{* *} \mathrm{E}\left(\mathrm{V}_{\mathrm{m}, \mathrm{l}}^{* *}\right)=$ $\mathrm{R}\left(\mathrm{x}_{\mathrm{E}}^{* *}\left(\mathrm{~V}_{\mathrm{m}, \mathrm{l}}^{* *}\right) \widehat{\theta_{\mathrm{m}}^{*}}\right)$ be the bootstrap pivotal quantities obtained from $\mathrm{x}^{*} \mathrm{E}\left(\mathrm{V}_{\mathrm{m}}^{*}\right)$ and $\mathrm{x}_{\mathrm{E}\left(\mathrm{V}_{\mathrm{m}, \mathrm{l}}^{* *}\right)}$, respectively with $\mathrm{B} 1$ and $\mathrm{B} 2$ being the number of first and second level bootstrap replications. Based on the above, we finally define the Monte Carlo algorithm for the iterated sufficient $\mathrm{m} / \mathrm{n}$ bootstrap for the construction of confidence sets

as follows.

(a) Resample $x_{E\left(V_{m}{ }^{*}\right)}$ from $x_{n}$ as explained above, and compute $R_{E\left(V_{m}{ }^{*}\right), i}$, for $\mathrm{i}=1, \ldots, B_{1}$.

(b) For each i, resample a second level bootstrap sample $x_{E\left(V_{m, l} l^{* *}\right)}$ from $x_{m}{ }^{*}$ and compute $R_{E\left(V_{m, l}^{* *}\right), j}$, for $\mathrm{j}=1, \ldots, B_{2}$.

(c) Calculate $Z_{i}=\frac{1}{B_{2}} \sum_{j=1}^{B_{2}} I\left(R^{* *} E\left(V_{\left.m, l^{* *}\right), j} \leq R^{*}{ }_{E\left(V_{m}\right.}{ }^{*}\right), i\right)$, for $\mathrm{i}=1, \ldots, B_{1}$.

(d) Then, the empirical cumulative distribution of Zi's, $G_{E\left(V_{m, l} l^{* *}\right)}^{*}$ is asymptotically $\mathrm{U}(0,1)$, and approximate the distribution of $R^{*}{ }_{E\left(V_{m}{ }^{*}\right)}, G^{*}{ }_{E\left(V_{m}{ }^{*}\right)}=P\left(x^{*}{ }_{E\left(V_{m}{ }^{*}\right)}, \widehat{\theta_{n}}\right)$.

(e) Define the level $\alpha$ confidence set of iterated sufficient $\mathrm{m} / \mathrm{n}$ bootstrap for $\mathrm{Rn}$

$$
\text { as } S_{E\left(V_{m, l^{* *}}\right)}^{* *}=\left\{\theta: R_{n}\left(x_{n}, \theta\right) \leq G_{E\left(V_{m}\right)^{*-1}}\left[G_{E\left(V_{m, l} l^{* *}\right.}{ }^{*-1}(\alpha)\right\}\right. \text {. }
$$

\section{Results}

In this section we consider the non-regular case described in Shao (1994) where the traditional and sufficient bootstrap methods fail to provide consistent results. First, in Section 3.1, we give the detailed information about this inconsistency problem and the behavior of sufficient $\mathrm{m} / \mathrm{n}$ bootstrap to establish this method's usefulness to avoid the vexing issue of inconsistency. A finite sample comparative study has been presented in Section 3.2.

\subsection{The Problem}

Let us consider the smooth function model described by Bhattacharya and Ghosh (1978). Suppose the data $X_{1}, \ldots, X_{n}$ are i.i.d. from $F$ with mean $\mu$. Let $\theta=g(\mu)$ with its estimate $\widehat{\theta_{n}}=g\left(\overline{X_{n}}\right)$, and further suppose that the asymptotic variance of $n^{1 / 2} \widehat{\theta_{n}}$ admits the representation $h(\mu)$ with the estimate $h\left(\overline{X_{n}}\right)$ for some smooth functions $g$ and $h$ on $R^{d}$. Suppose that $g$ and $h$ are continuously differentiable up to a sufficiently high order in an open neighborhood of $\mu$, and $\mathrm{F}$ satisfies Cramer's condition with sufficiently many finite moments. Let us assume that $\nabla \operatorname{gg}(\mu)=0$ and $\nabla^{2} \operatorname{g}(\mu) \neq 0$ where $\nabla \mathrm{g}$ is the gradient and $\nabla^{2} \mathrm{~g}$ is the Hessian matrix of $\mathrm{g}$. In this case instead of $\sqrt{\mathrm{n}}$-consistency, $\widehat{\theta_{\mathrm{n}}}$ is $\mathrm{n}$-consistent with a limiting distribution which approximates to a chi-squared type distribution. 
Babu (1984) shows that the traditional bootstrap fails to provide consistent estimator for the distribution of $R_{n}\left(x_{n}, \theta\right)$. Subsequently, this result can be extended in the case of the sufficient bootstrap since $\mathrm{E}\left(\mathrm{V}_{\mathrm{n}}{ }^{*}\right)=\mathrm{O}\left(1-\mathrm{e}^{-1}\right)$. The expansion of $\mathrm{E}\left(\mathrm{V}_{\mathrm{n}}{ }^{*}\right)\left(\hat{\theta}_{\mathrm{E}\left(\mathrm{V}_{\mathrm{n}}{ }^{*}\right)}-\hat{\theta}\right)$ can be written similar to the expansion of (2.4) given in Shao(1994) as

$$
\begin{gathered}
E\left(V_{n}{ }^{*}\right)\left(\widehat{\theta}_{E\left(V_{n}{ }^{*}\right)}-\hat{\theta}\right)=\nabla \mathrm{g}\left(\overline{X_{n}}\right)^{\prime}\left(\overline{X^{*}}{ }_{E\left(V^{*}{ }_{n}\right)}-\overline{X_{n}}\right)+\frac{1}{2}\left(\overline{X^{*}}{ }_{E\left(V^{*}{ }_{n}\right)}-\right. \\
\left.\overline{X_{n}}\right)^{\prime} \nabla^{2} \mathrm{~g}\left(\overline{X_{n}}\right)\left(\overline{X^{*}}{ }_{E\left(V^{*}{ }_{n}\right)}-\overline{X_{n}}\right)+o_{p}\left(E\left(V^{*-1}{ }_{n}\right)\right) \text { a.s. }
\end{gathered}
$$

Note that, the first term in Eq. 2 does not have an exact limit. It implies that the conditional distribution of $\mathrm{E}\left(\mathrm{V}_{\mathrm{n}}{ }^{*}\right)\left(\hat{\theta}_{\mathrm{E}\left(\mathrm{V}_{\mathrm{n}}{ }^{*}\right)}-\hat{\theta}\right)$ does not have a limit, suggesting that it is an inconsistent estimator of $G_{n}(x)$.On the other hand, it may be possible to regain the consistency with the iterated sufficient $\mathrm{m} / \mathrm{n}$ bootstrap by choosing $\mathrm{E}\left(\mathrm{V}_{\mathrm{m}}{ }^{*}\right)=\mathrm{o}(\mathrm{n})$ or $\mathrm{o}(\sqrt{\log \log \mathrm{n}})$.

In this non-regular case, Cheung et.al (2005) prove that both non-iterated and iterated $\mathrm{m} / \mathrm{n}$ bootstrap methods have the coverage error of order $\mathrm{O}\left(\mathrm{n}^{-1 / 2}\right)$ if $\mathrm{m}$ and $\mathrm{l}$ are chosen as $\mathrm{m}=\mathrm{o}(\mathrm{n})$ and $\mathrm{l}=$ $o(m)$. They further propose a new iterating scheme for the $\mathrm{m} / \mathrm{n}$ bootstrap to achieve the coverage error of order $\mathrm{O}\left(\mathrm{n}^{-2 / 3}\right)$ which can be further extended to our case of sufficient bootstrap through the following result.

Theorem. Let $\mathrm{K}(\mathrm{x}), \mathrm{H}(\mathrm{x}), \mathrm{D}(\beta), I_{m}(\alpha)$ and $J_{m . l}{ }^{*}(\alpha)$ be defined as in Cheung et.al (2005). Let also

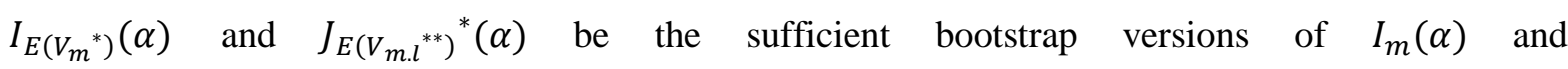
$J_{m . l}{ }^{*}(\alpha)$,respectively. Then, the asymptotic expansions of the coverage probabilities for non-iterated and iterated sufficient $\mathrm{m} / \mathrm{n}$ bootstrap confidence limits are obtained as follows.

(a) For $m=o(n)$ and $m \rightarrow \infty$,

$$
\begin{aligned}
\boldsymbol{P}_{\boldsymbol{F}}\left(\theta \leq I_{E\left(V_{m}\right)}\right) & (\alpha)) \\
& =\alpha-E\left(V_{n}{ }^{*}\right) n^{-1} D(1-\alpha)+E\left(V_{m}{ }^{*}\right)^{-1} K\left(H^{-1}(1-\alpha)\right)+O\left(E\left(V_{m}{ }^{*}\right)^{-2}\right. \\
& \left.+E\left(V_{m}{ }^{*}\right)^{*} n^{-2}\right) \\
& =\alpha-\frac{m}{n}(1+o(1)) D(1-\alpha)+\frac{1}{m}(1+o(1))^{-1} K\left(H^{-1}(1-\alpha)\right)+O((1 \\
& \left.+o(1))^{-2}+(1+o(1))^{2}\right) .
\end{aligned}
$$


(b) For $m=o(n), l=o(m)$ and $l \rightarrow \infty$,

$$
\begin{aligned}
& \boldsymbol{P}_{\boldsymbol{F}}\left(\theta \leq J_{E\left(V_{m, l}^{* *}\right)}^{*}(\alpha)\right) \\
&=\alpha-\left(2 E\left(V_{m}^{*}\right) n^{-1}-E\left(V_{m, l}{ }^{* *}\right) E\left(V_{m}{ }^{*}\right)^{-1} D(1-\alpha)\right. \\
&+ E\left(V_{m, l}{ }^{* *}\right)^{-1} K\left(H^{-1}(1-\alpha)\right)+O\left(E\left(V_{m}^{*}\right)^{2} n^{-2}+E\left(V_{m, l}{ }^{* *}\right)^{-2}\right. \\
&\left.+E\left(V_{m, l}{ }^{* *}\right)^{2} E\left(V_{m}^{*}\right)^{-2}\right) \\
&=\alpha-\left(2 \frac{m}{n}(1+o(1))-\frac{l}{m}(1+o(1))\right) D(1-\alpha)+\frac{1}{l}(1 \\
&+o(1))^{-1} K\left(H^{-1}(1-\alpha)\right)+O\left((1+o(1))^{2}+(1+o(1))^{-2}+(1+o(1))^{2}\right)
\end{aligned}
$$

Proof. By Taylor series expansion and using the fact that $E\left(V_{m}{ }^{*}\right)=n\left(1-e^{-m / n}\right)$ and $E\left(V_{m, l}{ }^{* *}\right)=$ $m\left(1-e^{-l / m}\right)$ the proof of Theorem directly follows from Proposition 1 as in Cheung et.al (2005).

\subsection{A numerical study}

We consider the exponential example given by Cheung et.al (2005) to compare the coverage performances of $\mathrm{m} / \mathrm{n}$, sufficient $\mathrm{m} / \mathrm{n}$ bootstrap methods and their iterated versions. The underlying distribution $\mathrm{F}$ is chosen to be Normal $\mathrm{N}(0, \Sigma)$ where $\Sigma$ is defined as in Cheung et.al (2005), and the parameter of interest is $\theta=\mathrm{g}(\mu)=\exp \left(\|\mu\|^{2}\right)$. We estimate the coverage errors for $\alpha$ level upper confidence interval for $\theta$ using 2000 simulations for $\alpha 0.05,0.1,0.5,0.90$ and 0.95 , with $\mathrm{n}=1000$, 5000, 10000, 15000 and 20000, $\mathrm{m}=n^{1 / 2}, l=\frac{2 m^{2}}{n}$, and $B_{1}=B_{2}=1000$. The results are presented in Figure 1. Generally, the errors obtained from the large $\alpha$ values seem to be smaller than the ones for small $\alpha$ values. For $\alpha=0.05$ and 0.1 , with increasing n, the coverage accuracies of the percentile confidence intervals get better for both traditional and iterated sufficient $\mathrm{m} / \mathrm{n}$ bootstrap methods. On the other hand, the errors obtained from the non-iterated $\mathrm{m} / \mathrm{n}$ versions remain the same. For $\alpha=0.5$, while the iterated methods have better performances compared to non-iterated versions in small samples, the coverage accuracy of the methods tend to be similar in large sample sizes. For $\alpha=$ 0.90 and 0.95 , all the methods have similar performances.

We also consider the $\mathrm{m}$ in the form of $\mathrm{m} \propto \mathrm{C}_{\mathrm{n}^{1 / 2}}$ to evaluate the effect of $C$. We have chosen the $C$ values $C=0.5,1,1.5,2,3$ and, 5 for two fixed sample sizes: $\mathrm{n}=1000$ and $\mathrm{n}=10000$. The results are presented in Figures 2 and3, respectively. For smaller $\alpha$ values, it seems that the coverage accuracies of the non-iterated bootstrap methods do not get affected by the $C$ values. For moderate and large $\alpha$ values, with moderate $C$ values such as $C=2$ or $C=3$, we get smaller errors. 


\section{Conclusion}

In this study, we propose introducing the iterating principle in the sufficient $\mathrm{m} / \mathrm{n}$ bootstrap method to improve the coverage accuracy of bootstrap percentile confidence intervals in non-regular smooth function models. The results of simulation study show that similar or even better coverage accuracy of iterated $\mathrm{m} / \mathrm{n}$ bootstrap can be obtained by using iterated sufficient $\mathrm{m} / \mathrm{n}$ bootstrap method. The iteration increases the computing time since double bootstrap is used. On the other hand, using only distinct units in resamples will reduce the computational burden which is an important result of this study. Our records (only for one simulation) show that the computing times are roughly $3.36 \mathrm{~min}$ for iterated $\mathrm{m} / \mathrm{n}$ bootstrap and $2.56 \mathrm{~min}$ for the sufficient bootstrap version, respectively, when B1 = B2 = 1000 and $\mathrm{n}=$ 20000. That is, by using sufficient bootstrap the computational burden of iterated $\mathrm{m} / \mathrm{n}$ bootstrap can be reduced roughly by $24 \%$.

As a future study, the performance of the proposed method can also be stud-ied studied for testing mixture models or for examining the symmetry of the underlying distribution as an alternative to the proposed methods, respectively by Yang et al. (2010) and Zheng and Gastwirth (2010). The proposed method can also be used in time series data as studied by Beyaztas and Firuzan (2016).

\section{Acknowledgements}

We thank an anonymous referee whose comments/suggestions helped improve and clarify this manuscript. Ufuk Beyaztas was supported by a grant from the Scientific and Technological Research Council of Turkey (TUBITAK) grant no: 1059B141500288. Soutir Bandyopadhyay's work has been partially supported by NSF-DMS 1406622. 

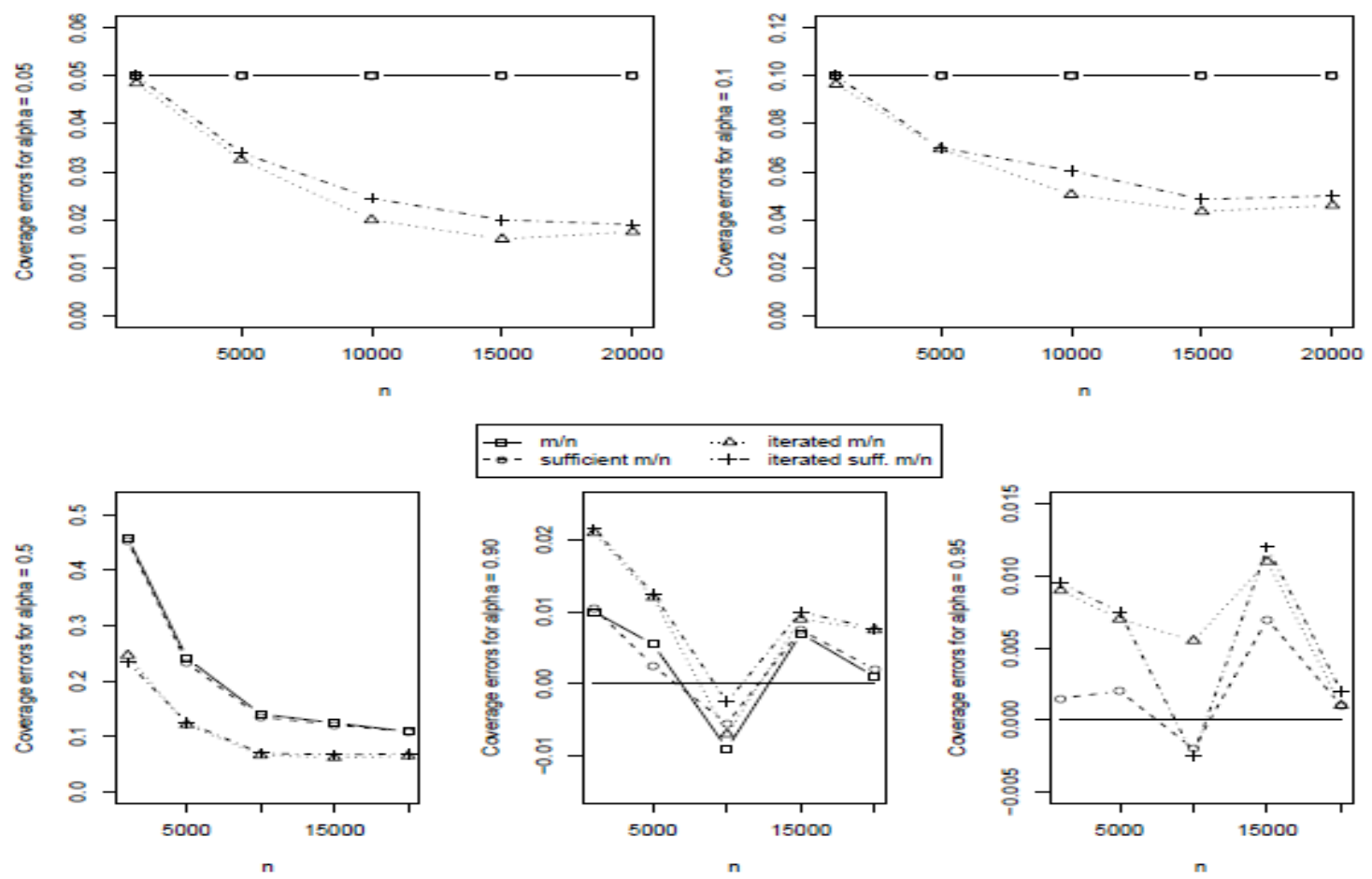

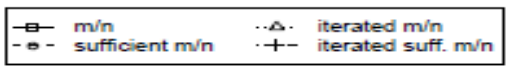
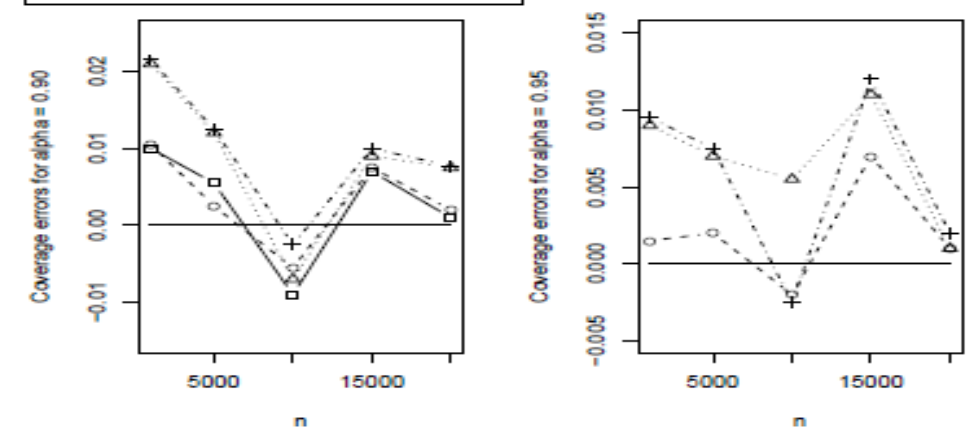

Figure 1: Coverage performances of the bootstrap methods
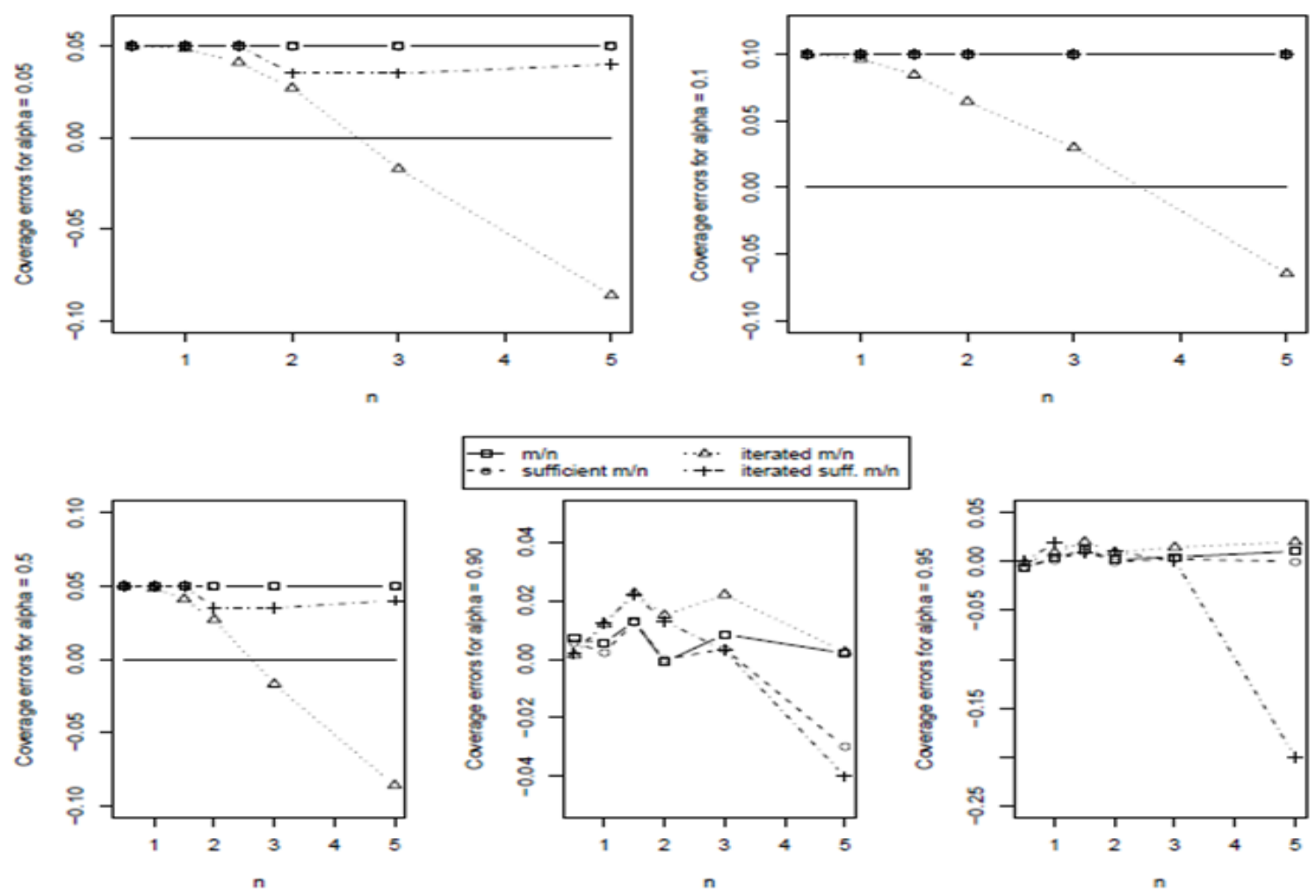

Figure 2: Coverage performances of the bootstrap methods according to $C$ values when $n=1000$ 

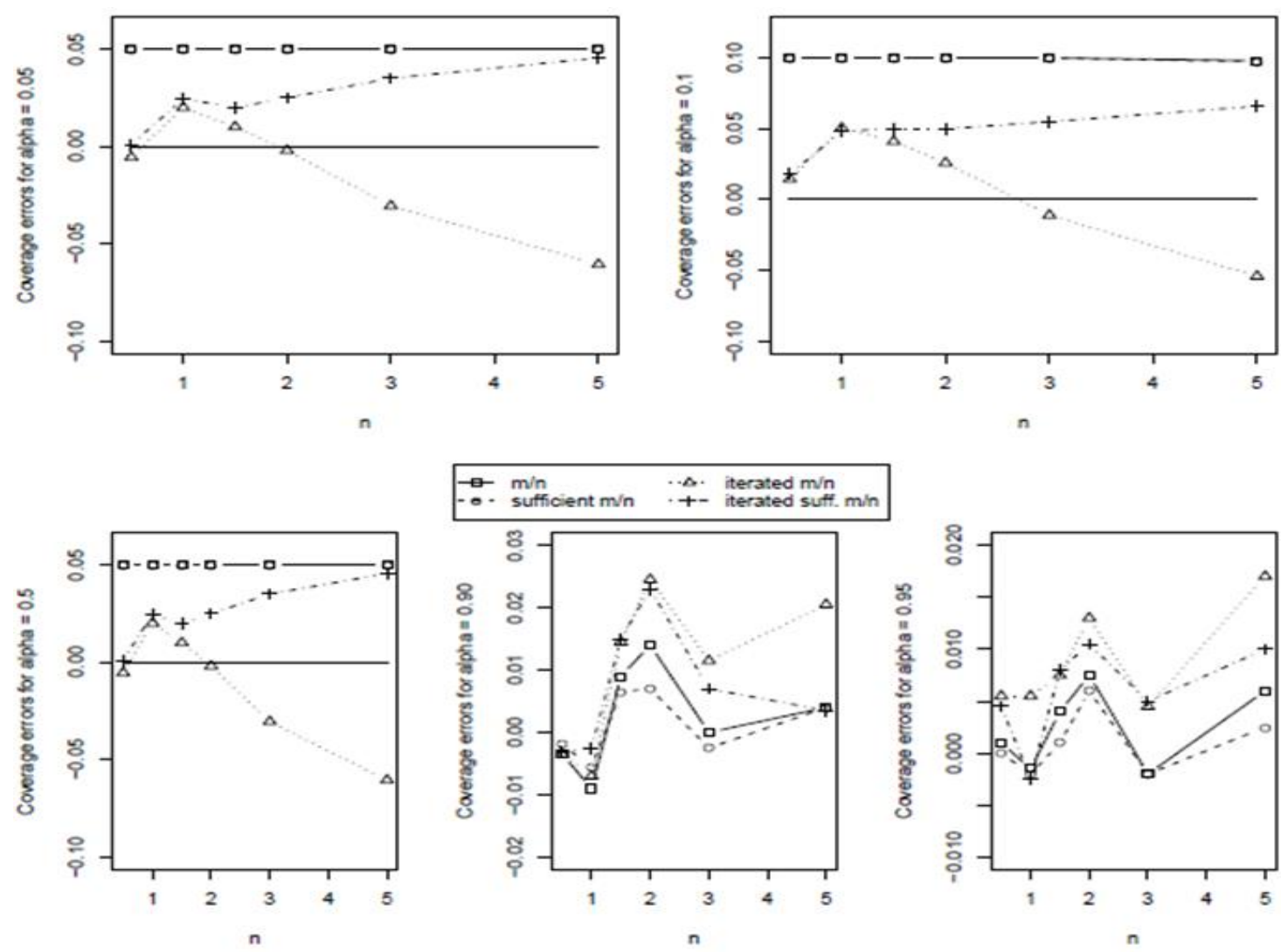

Figure 3: Coverage performances of the bootstrap methods according to $C$ values when $n=10000$ 


\section{References}

[1] Alin, A., Martin, M. A., Beyaztas, U., and Pathak, P. K. (2017). Sufficient m-out-of-n (m/n) bootstrap. Journal of Statistical Computation and Simulation, 87, 1742-1753.

[2] Atherya, K. B. (1978). Bootstrap of the mean in the infinite variance case.Annals of Statistics, 15, 724-731.

[3] Babu, G. J. (1984). Bootstrapping statistics with linear combinations of chi- square as weak limit. Sankhya, 46, 58-93.

[4] Beran, R. (1987). Prepivoting to reduce level error of confidence sets. Biometrika,74, 151-173.

[5] Beran, R. (1997). Diagnosing bootstrap success. Annals of the Institute of Statistical Mathematics, $49,1-24$.

[6] Beyaztas, B. H., and Firuzan, E. (2016). An empirical comparison of block bootstrap methods: traditional and newer ones. Journal of Data Science, 14, 641-656.

[7] U. Beyaztas, A. Alin, S. Bandyopadhyay 11 Bhattacharya, R. N., and Ghosh, J. K. (1978). On the validity of the formal Edgeworth expansion. Annals of Statistics, 6, 434-451.

[8] Bickel, P. J., and Freedman, D. A. (1981). Some asymptotic theory for the bootstrap. Annals of Statistics, 9, 1196-1217.

[9] Bretagnolle, J. (1983). Lois limites du bootstrap de certaines fonctionnelles. Annales de l'Institute Henri Poincare, Section B,, 19, 291-296.

[10] Efron, B. (1979). Another look at the jackknife. Anals of Statistics, 7, 1-26.

[11] Cheung, K. Y., Lee., S. M. S., and Young, G. A. (2005). Iterating the mout of $n$ bootstrap in non-regular smooth functional models. Statistica Sinica, 15, 945-967.

[12] DiCiccio, T. J., and Romano, J.P. (1988). A Review of Bootstrap Confidence In- tervals. Journal of The Royal Statistical Society Series B-statistical Method- ology, 50, 338-354.

[13] Hall, P. (1986). On the bootstrap and confidence intervals. Annals of Statistics,54, 1431-1452.

[14] Hall, P., and Martin, M. A. (1988). On bootstrap resampling and iteration.Biometrika, 75, 661-671. 
[15] Martin, M. A. (1990). On bootstrap iteration for coverage correction in confidence intervals. Journal of the American Statistical Association, 85, 1105-1118.

[16] Shao, J. (1994). Bootstrap sample size in non-regular cases. Proceedings of the American Mathematical Society, 122, 1251-1262.

[17] Singh, S., and Sedory, S. A. (2011). Sufficient bootstrapping. Computational Statistics and Data Analysis, 55, 1629-1637.

[18] Swanepoel, J. W. H. (1986). A note on proving that the (modified) bootstrap works. Communications in Statistics - Theory and Methods, 15, 3193-3203.

[19] Wei, B., Lee, S., and Wu, X. (2014). Stochastically optimal bootstrap sample size for shrinkage-type statistics. Statistics and Computing, 26, 249-262.

[20] Yang, Y., Fan, J., and May S. (2010). Bimodality of Plasma Glucose Distributions in Whites: A Bootstrap Approach to Testing Mixture Models. Journal of Data Science, 8, 483-493.

[21] Zheng, T., and Gastwirth, J. (2010). On bootstrap test of symmetry about an unknown median. Journal of Data Science, 8, 397-412. 

FUNCTION MODELS

Ufuk Beyaztas

Department of Statistics

Bartin University Bartin, 74100, Turkey

Aylin Alin

Department of Statistics

Dokuz Eylul University

Izmir, 35160, Turkey

Soutir Bandyopadhyay

Department of Applied Mathematics and Statistics

Colorado School of Mines

Golden, 80401, CO, USA 